\title{
A STUDY ON ABSOLUTE SUMMABILITY \\ FACTORS FOR A TRIANGULAR MATRIX
}

\author{
EKREM SAVAŞ
}

Abstract. In this paper we obtain an absolute summability factor theorem for lower triangular matrices.

Mathematics subject classification (2000): 40F05, 40D25.

Keywords and phrases: Absolute summability, summability factors.

\section{REFERENCES}

[1] B. E. RHOADES, Inclusion theorems for absolute matrix summability methods, J. Math. Anal. Appl. 238(1999), 82-90.

[2] W. T. Sulaiman, A study relation between two summability methods, Proc. American Math. Soc. 115(1992), 303-312. 